\section{Leaky guts: intestinal permeability and NASH}

New research shows that NASH is associated with increased intestinal permeability and indicates that an early phase of liver injury and inflammation contributes to this breach in the intestinal barrier. The findings add weight to the concept of gut-liver crosstalk in disease. "Emerging data link increases in intestinal permeability to a variety of nonintestinal disorders," note authors Raymond Chung and Suraj Patel. "The idea that a leaky gut can cause problems outside the intestine relies on the hypothesis that microbial products in the intestine are able to escape the gut and elicit an inflammatory reaction wherever they may end up."

Chung, Patel and colleagues took a two-pronged approach in their study: a meta-analysis of the existing literature to compare the rates of increased intestinal permeability in patients with or without NAFLD, and in vitro and in vivo experiments to explore the underlying mechanisms that contribute to this change in intestinal permeability.

According to their meta-analysis, $39.1 \%$ of patients with NAFLD had evidence of increased intestinal permeability compared with only $6.8 \%$ of healthy individuals as controls, with an OR of 5.08 (95\% CI 1.98-13.05). A subgroup analysis revealed that patients with NASH had a higher incidence of changes to intestinal permeability than patients with NAFLD (including those with bland steatosis) and were more likely to have increased intestinal permeability than healthy individuals (OR 7.21; 95\% CI 2.34-22.13).

The relationship between intestinal permeability and NAFLD was explored in a diet-induced mouse model of NASH (whereby mice are fed a diet deficient in methionine and choline, the MCD diet). Mice fed this MCD diet developed liver injury within 6 days, which corresponded with increased serum levels of alanine aminotransferase (ALT) and hepatic and systemic levels of TNF as well as histological evidence of hepatic steatosis and inflammation. In a secondary liver injury phase, increased serum ALT levels, NAFLD activity scores and TNF levels peaked at day 21. Notably, a marked increase in intestinal permeability was not observed until day 10 , which coincided with evidence of disruption of localization of the tight junction protein $\mathrm{ZO}-1$ in the small intestine (indicative of injury to the epithelial tight junction complex).

Interestingly, in vitro experiments demonstrated that MCD media induced injury and inflammation when applied directly to hepatocytes and Kupffer cells, but had no negative effects on intestinal epithelial cells. Furthermore, mice resistant to acute TNF-induced intestinal barrier loss in experimental IBD still developed MCD-diet-induced liver injury and notable intestinal permeability, indicating that TNF does not have a dominant role in the changes observed.

The investigators postulate that increased intestinal permeability is an effect of rather than a cause of NASH. They plan further work to "find a soluble protein produced in the liver in response to early fat accumulation and injury that has the potential to alter intestinal permeability and contribute to ongoing liver injury and inflammation".

\section{Katrina Ray}

Original article Luther, J. et al. Hepatic injury in non-alcoholic steatohepatitis contributes to altered intestinal permeability. Cell. Mol. Gastroenterol. Hepatol. doi:10.1016/jchmgh.2015.01.001

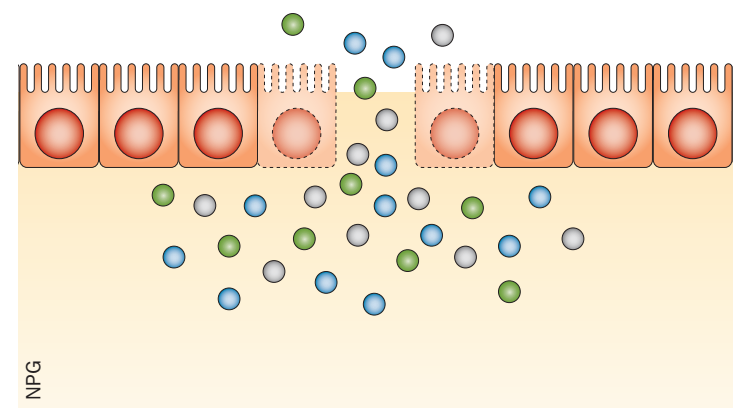

